\title{
ON THE CONSTRUCTION OF PARTIAL DIFFERENCE SCHEMES II: DISCRETE VARIABLES AND SCHWARZIAN LATTICES
}

\author{
Decio Levi ${ }^{a, *}$, Miguel A. Rodríguez ${ }^{b}$ \\ ${ }^{a}$ Dipartimento di Matematica e Fisica, Università degli Studi Roma Tre and INFN Sezione di Roma Tre, Via \\ della Vasca Navale 84, 00146 Roma, Italy \\ ${ }^{b}$ Departamento de Física Teórica II, Facultad de Físicas, Universidad Complutense, 28040 Madrid, Spain \\ * corresponding author: decio.levi@roma3.infn.it
}

\begin{abstract}
In the process of constructing invariant difference schemes which approximate partial differential equations we write down a procedure for discretizing a partial differential equation on an arbitrary lattice. An open problem is the meaning of a lattice which does not satisfy the ClairautSchwarz-Young theorem. To analyze it we apply the procedure on a simple example, the potential Burgers equation with two different lattices, an orthogonal lattice which is invariant under the symmetries of the equation and satisfies the commutativity of the partial difference operators and an exponential lattice which is not invariant and does not satisfy the Clairaut-Schwarz-Young theorem. A discussion on the numerical results is presented showing the different behavior of both schemes for two different exact solutions and their numerical approximations.
\end{abstract}

KEYWORDs: partial differential and difference equations; discretization; Clairaut-Schwarz-Young theorem.

AMS Mathematics Subject Classification: 39A14, 35F05.

\section{IntRoduCtion}

The construction of difference equations, written as invariants of the continuous group of symmetries of differential equations, is part of a project to apply symmetry group methods to the numerical solution of differential equations [2, 3, 6, 9, 11, 13, 15, 17, 19, 23. This project has accomplished a significative advance since its first introduction last century. In particular, the construction of invariant schemes has proven to be a very fruitful approach in the construction of numerical schemes for ordinary differential equations [2, 3], in cases when the usual approaches present serious problems of convergence and accuracy, for instance, in the behavior of the solutions in the neighborhood of a singularity. In this problem a deeper understanding of the mathematics involved in its relation with numerics can provide results important for their applications to problems in Physics and Mathematics.

The usual procedure in this framework is to compute the symmetry group of the differential equation and then compute the invariant lattice and invariant difference equation with respect to the symmetry group. However, since the differential and difference calculus present substantial differences, a special care has to be taken to assure the consistency of the approach. For example, the Clairaut-Schwarz-Young theorem on the equality of the cross derivatives, which is satisfied in the continuous case under some mild conditions on the functions, is not valid in the discrete case for a general lattice. Recently it has been shown 14 that the discrete Clairaut-Schwarz-Young theorem, equality of the cross differences, imposes strong restrictions on the lattice. Moreover, the construction of the discrete invariant scheme starting from the discrete invariants is not at all obvious as it is usually obtained by finding a proper combination of the continuous limits of the various discrete invariants. The main idea guiding the construction of the whole scheme, that is the difference equations and the equations defining the lattice, which in some cases are mixed, is that the continuous limit yields the differential equation and trivial identities.

This article is a continuation of our work on the construction of partial difference schemes [14]. In the previous work we concentrated on the Clairaut-Schwarz-Young theorem. Here we introduce by a one to one correspondence a new set of discrete coordinates which describe the partial difference equation on the lattice. In terms of these coordinate systems we can write immediately the discrete counterpart of any continuous invariant. So we can discretize in a straightforward way any partial differential equation described in terms of the invariants of a group of symmetries.

A particular role in the construction of discrete invariant schemes is played by the lattice. Consequently the Clairaut-Schwarz-Young theorem can play an important role in discriminating lattice schemes, i.e., the combination of the discrete equation and its lattice.

In Section 2 we study schemes for scalar partial differential equations and show the constraints on the group transformations due to the Clairaut-Schwarz-Young theorem. Using these results, in Section 3 we construct 
in a standard way the invariant discrete potential Burgers and in Section 4. we study it numerically for two different lattices, one invariant and Schwarzian and one not, for two different exact solutions of the differential equation. Some concluding remarks are presented in Section 5.

\section{SCHEMES FOR PARTIAL DIFFERENCE EQUATIONS}

In the case of ordinary difference equations of order $K$ for one dependent variable $u_{n}$ and one independent variable $x_{n}$, a natural scheme is given by the points $\left\{x_{n+k-1}, u_{n+k-1}, 1 \leq k \leq K+1\right\}$ for some fixed $n$. An alternative equivalent set of coordinates on the scheme is given by [14, 23]:

$$
\left\{x_{n}, u_{n}, p_{n+1}^{(1)}, p_{n+2}^{(2)}, p_{n+3}^{(3)}, p_{n+4}^{(4)}, \ldots p_{n+K-1}^{(K-1)}, p_{n+K}^{(K)}, h_{n+1}, h_{n+2}, \ldots h_{n+K}\right\}
$$

with

$$
\begin{gathered}
h_{n+k}=x_{n+k}-x_{n+k-1}, \quad 1 \leq k \leq K, \\
p_{n+k}^{(k)}=\left[D_{x}\right]^{k} u_{n}, \quad \text { where } \quad D_{x}=\frac{1}{h_{n+1}}\left[T_{x}-1\right], \quad T_{x} u_{n}=u_{n+1}, \quad k \in \mathbb{Z}^{+}
\end{gathered}
$$

In the continuous limit $h_{n+k} \rightarrow 0$ and $p_{n+k}^{(k)} \rightarrow \frac{d^{k} u(x)}{d x^{k}}$. If we transform the standard discrete prolongation [17]

$$
\operatorname{pr}^{(K)} \hat{X}=\sum_{k=n}^{n+K}\left(\xi_{k}\left(x_{k}, u_{k}\right) \partial_{x_{k}}+\phi_{k}\left(x_{k}, u_{k}\right) \partial_{u_{k}}\right)
$$

of the vector field

$$
\hat{X}=\xi_{n}\left(x_{n}, u_{n}\right) \partial_{x_{n}}+\phi_{n}\left(x_{n}, u_{n}\right) \partial_{u_{n}}
$$

to the new variables (1), we obtain

$$
\operatorname{pr} \hat{X}=\xi_{n}\left(x_{n}, u_{n}\right) \partial_{x_{n}}+\phi_{n}\left(x_{n}, u_{n}\right) \partial_{u_{n}}+\sum_{k=1}^{K} \kappa^{(k)} \partial_{h_{n+k}}+\sum_{k=1}^{K} \phi_{n+k}^{(k)} \partial_{p_{n+k}^{(k)}} .
$$

The general formulas for the coefficients $\kappa^{(k)}$ and $\phi^{(k)}$ are

$$
\begin{gathered}
\kappa^{(k)}=\xi_{n+k}-\xi_{n+k-1}, \\
\phi_{n+k}^{(k)}=D_{x} \phi_{n+k-1}^{(k-1)}-p_{n+k}^{(k)} D_{x} \xi_{n}, \quad k=1,2, \ldots, K .
\end{gathered}
$$

It is worthwhile to notice that both the higher order discrete derivatives and their corresponding prolongations are written in terms of $x_{n}, u_{n}, \xi_{n}\left(x_{n}, u_{n}\right), \phi_{n}\left(x_{n}, u_{n}\right)$ and their difference consequences obtained by applying the operator $D_{x}$ given in (2). This way of constructing invariant ordinary difference equation is different from that considered in 13 but is easily extendible to the case of partial difference equations.

For partial difference equations we consider here only, for simplicity, the case of one dependent variable and two independent variables as will be the example we will discuss in the following Section. Moreover, as we will deal with a nonlinear partial difference equation of second order we limit ourselves to a scheme of six points $(n, m),(n+1, m),(n, m+1),(n+2, m),(n, m+2),(n+1, m+1)$, the minimum number of points necessary to get all partial second derivatives as first order approximations. The variables $x, y$ and $u(x, y)$ in all points correspond to 18 data, 12 related to the independent variables and 6 to the dependent one. The extension to more variables and higher order equations requires just more points but it is straightforward. Having 12 data for the independent variables we can construct from them 10 differences

$$
\begin{array}{rlll}
x_{0,0}, \quad y_{0,0}, \quad & h_{0,0}^{x}=x_{1,0}-x_{0,0}, \quad h_{0,0}^{y}=y_{0,1}-y_{0,0}, \quad \sigma_{0,0}^{x}=x_{0,1}-x_{0,0}, \quad \sigma_{0,0}^{y}=y_{1,0}-y_{0,0}, \\
& h_{1,0}^{x}=x_{2,0}-x_{1,0}, \quad h_{0,1}^{y}=y_{0,2}-y_{0,1}, \quad \sigma_{0,1}^{x}=x_{0,2}-x_{0,1}, \quad \sigma_{1,0}^{y}=y_{2,0}-y_{1,0}, \\
& h_{0,1}^{x}=x_{1,1}-x_{0,1}, \quad h_{1,0}^{y}=y_{1,1}-y_{1,0},
\end{array}
$$

where for convenience of notation here and in the following, whenever it may not create misunderstanding, we have indicated just the distance from the values $n, m$ of the indices. From the values of the dependent variables in the 6 points we can calculate the 6 quantities

$$
u_{0,0}, \quad D_{x} u_{0,0}, \quad D_{y} u_{0,0}, \quad\left[D_{x}\right]^{2} u_{0,0}, \quad\left[D_{y}\right]^{2} u_{0,0}, \quad D_{x} D_{y} u_{0,0},
$$

where the operators $D_{x}$ and $D_{y}$, introduced in [14], are given by

$$
D_{x}=\frac{1}{h_{0,0}^{x} h_{0,0}^{y}-\sigma_{0,0}^{x} \sigma_{0,0}^{y}}\left(h_{0,0}^{y} \Delta_{n}-\sigma_{0,0}^{y} \Delta_{m}\right), \quad D_{y}=\frac{1}{h_{0,0}^{x} h_{0,0}^{y}-\sigma_{0,0}^{x} \sigma_{0,0}^{y}}\left(-\sigma_{0,0}^{x} \Delta_{n}+h_{0,0}^{x} \Delta_{m}\right)
$$


with

$$
T_{n} f_{n, m}=f_{n+1, m}, \quad \Delta_{n}=T_{n}-1, \quad T_{m} f_{n, m}=f_{n, m+1}, \quad \Delta_{m}=T_{m}-1 .
$$

These operators are the discrete counterpart of the partial derivatives in the $x, y$ axes directions, written in terms of the partial difference operators in the lattice directions, which, generically, do not coincide with the cartesian axes. See [14 for a more detailed description.

Note that $D_{y} D_{x} u_{0,0}$ is not independent from the 6 quantities (8). It can be written in term of (7) and (8). For a generic lattice we have:

$$
\begin{aligned}
& D_{y} D_{x} u_{0,0}=-D_{x} D_{x} u_{0,0} \frac{\left(h_{0,0}^{x}-h_{0,1}^{x}\right)\left(h_{0,0}^{x}-\sigma_{0,0}^{x}\right)}{h_{0,1}^{x} h_{0,0}^{y}+h_{0,0}^{x} \sigma_{0,0}^{y}-h_{0,1}^{x} \sigma_{0,0}^{y}-\sigma_{0,0}^{x} \sigma_{0,0}^{y}}\left(h_{0,0}^{y}-h_{1,0}^{y}\right)\left(h_{0,0}^{y}-\sigma_{0,0}^{y}\right) \\
&+D_{y} D_{y} u_{0,0} \frac{\left(h_{0,1}^{x} h_{0,0}^{y}+h_{0,0}^{x} \sigma_{0,0}^{y}-h_{0,1}^{x} \sigma_{0,0}^{y}-\sigma_{0,0}^{x} \sigma_{0,0}^{y}\right.}{h_{0,0}^{x}} \\
& \quad+D_{x} D_{y} u_{0,0} \frac{h_{0,0}^{x} h_{1,0}^{y}+h_{0,0}^{y} \sigma_{0,0}^{x}-h_{1,0}^{y} \sigma_{0,0}^{x}-\sigma_{0,0}^{x} \sigma_{0,0}^{y}}{h_{0,1}^{x} h_{0,0}^{y}+h_{0,0}^{x} \sigma_{0,0}^{y}-h_{0,1}^{x} \sigma_{0,0}^{y}-\sigma_{0,0}^{x} \sigma_{0,0}^{y}}
\end{aligned}
$$

Formulas (7)-10 can be simplified if we require the validity of the Clairaut-Schwarz-Young theorem, i.e., $D_{x} D_{y} u_{0,0}=D_{y} D_{x} u_{0,0}$. This is true when the following constraint for the lattice holds [14]:

$$
\sigma_{n, m}^{x}=\sigma_{n+1, m}^{x} \equiv \sigma_{m}^{x}, \quad h_{n, m}^{x}=h_{n, m+1}^{x} \equiv h_{n}^{x}, \quad \sigma_{n, m}^{y}=\sigma_{n, m+1}^{y} \equiv \sigma_{n}^{y}, \quad h_{n, m}^{y}=h_{n+1, m}^{y} \equiv h_{m}^{y} .
$$

Using the operators $D_{x}$ and $D_{y}$ given in $[9]$ we can transform the standard discrete prolongation [17.

$$
\operatorname{pr} \hat{X}_{n, m}=\hat{X}_{n, m}+\hat{X}_{n+1, m}+\hat{X}_{n+2, m}+\hat{X}_{n, m+1}+\hat{X}_{n, m+2}+\hat{X}_{n+1, m+1}
$$

of the vector field

$$
\hat{X}_{n, m}=\xi_{n, m} \partial_{x_{n, m}}+\tau_{n, m} \partial_{y_{n, m}}+\phi_{n, m} \partial_{u_{n, m}}
$$

to the new set of independent variables (7), (8). We get

$$
\begin{aligned}
\operatorname{pr} \hat{X}_{n, m} & =\hat{X}_{n, m} \\
& +\sum_{(i, j)=0,1}\left[\eta_{n+i, m+j}^{(x)} \partial_{h_{n+i, m+j}^{x}}+\chi_{n+i, m+j}^{(x)} \partial_{\sigma_{n+i, m+j}^{x}}+\eta_{n+i, m+j}^{(y)} \partial_{h_{n+i, m+j}^{y}}+\chi_{n+i, m+j}^{(y)} \partial_{\sigma_{n+i, m+j}^{y}}\right] \\
& \quad+\phi_{n, m}^{(1, x)} \partial_{D_{x} u_{n, m}}+\phi_{n, m}^{(1, y)} \partial_{D_{y} u_{n, m}}+\phi_{n, m}^{(2, x x)} \partial_{\left[D_{x}\right]^{2} u_{n, m}}+\phi_{n, m}^{(2, x y)} \partial_{D_{y} D_{x} u_{n, m}}+\phi_{n, m}^{(2, y y)} \partial_{\left[D_{y}\right]^{2} u_{n, m}}
\end{aligned}
$$

where

$$
\begin{gathered}
\eta_{n+i, m+j}^{(x)}=\xi_{n+1+i, m+j}-\xi_{n+i, m+j}, \quad \eta_{n+i, m+j}^{(y)}=\tau_{n+i, m+1+j}-\tau_{n+i, m+j}, \\
\chi_{n+i, m+j}^{(x)}=\xi_{n+i, m+1+j}-\xi_{n+i, m+j}, \quad \chi_{n+i, m+j}^{(y)}=\tau_{n+1+i, m+j}-\tau_{n+i, m+j}, \\
\phi_{n, m}^{(1, x)}=D_{x} \phi_{n, m}-D_{x} u_{n, m} D_{x} \xi_{n, m}-D_{y} u_{n, m} D_{x} \tau_{n, m}, \\
\phi_{n, m}^{(1, y)}=D_{y} \phi_{n, m}-D_{x} u_{n, m} D_{y} \xi_{n, m}-D_{y} u_{n, m} D_{y} \tau_{n, m}, \\
\phi_{n, m}^{(2, x x)}=D_{x} \phi_{n, m}^{(1, x)}-\left[D_{x}\right]^{2} u_{n, m} D_{x} \xi_{n, m}-D_{y} D_{x} u_{n, m} D_{x} \tau_{n, m}, \\
\phi_{n, m}^{(2, x y)}=D_{x} \phi_{n, m}^{(1, y)}-D_{x} D_{y} u_{n, m} D_{x} \xi_{n, m}-\left[D_{y}\right]^{2} u_{n, m} D_{x} \tau_{n, m}, \\
\phi_{n, m}^{(2, y y)}=D_{y} \phi_{n, m}^{(1, y)}-D_{x} D_{y} u_{n, m} D_{y} \xi_{n, m}-\left[D_{y}\right]^{2} u_{n, m} D_{y} \tau_{n, m} .
\end{gathered}
$$

In the continuous limit, when $h_{n+i, m+j}^{x}, h_{n+i, m+j}^{y}, \sigma_{n+i, m+j}^{x}$ and $\sigma_{n+i, m+j}^{y}$ go to $0, \eta_{n+i, m+j}^{(x)}, \eta_{n+i, m+j}^{(y)}, \chi_{n+i, m+j}^{(x)}$ and $\chi_{n+i, m+j}^{(y)}$ go also to 0 while $\phi_{n, m}^{(1, x)}, \phi_{n, m}^{(1, y)}, \phi_{n, m}^{(2, x x)}, \phi_{n, m}^{(2, x y)}$ and $\phi_{n, m}^{(2, y y)}$ go to the corresponding continuous prolongations.

Applying the infinitesimal generator (14) onto 11) we get that both functions $\xi_{n, m}\left(x_{n, m}, y_{n, m}, u_{n, m}\right)$ and $\tau_{n, m}\left(x_{n, m}, y_{n, m}, u_{n, m}\right)$ must satisfy the discrete wave equations

$$
\xi_{n, m+1}-\xi_{n, m}-\xi_{n+1, m+1}+\xi_{n+1, m}=0, \quad \tau_{n, m+1}-\tau_{n, m}-\tau_{n+1, m+1}+\tau_{n+1, m}=0 .
$$

This is a constraint for the symmetry coefficients if the Clairaut-Schwarz-Young theorem is to be satisfied, i.e., (16) are to be added to the determining equations if we want to have a lattice satisfying the ClairautSchwarz-Young theorem. In this case, if, for example, the difference equation for $u_{n, m}$ involves second order shifts like $u_{n+2, m}$ or $u_{n, m+2}$ so that $u_{n, m}, u_{n, m+1}$ and $u_{n+1, m}$ are independent variables, then from (16) we get $\xi_{n, m}\left(x_{n, m}, y_{n, m}, u_{n, m}\right)=\xi_{n, m}\left(x_{n, m}, y_{n, m}\right)$. If the lattice equations in our scheme involve the points $x_{n+2, m}, y_{n+2, m}$ or $x_{n, m+2}, y_{n, m+2}$, so that $x_{n, m}, x_{n, m+1}, x_{n+1, m}, y_{n, m}, y_{n, m+1}$ and $y_{n+1, m}$ are independent variables, then $\xi_{n, m}\left(x_{n, m}, y_{n, m}, u_{n, m}\right)=\xi_{n, m}$. Then, as it is the case of the continuous wave equation, the 
general solution of 16 is given by $\xi_{n, m}=f_{n}^{(x)}+g_{m}^{(x)}$ and $\tau_{n, m}=f_{n}^{(y)}+g_{m}^{(y)}$, i.e., the sum of an arbitrary function of $n$ and one of $m$.

It is worthwhile, in view of the application to be carried out in next Section, to compute the lowest order discrete derivatives of monomials in $x$ and $y$ :

$$
\begin{gathered}
D_{x} x_{n, m}=1, \quad D_{x} y_{n, m}=0, \quad D_{y} x_{n, m}=0, \quad D_{y} y_{n, m}=1, \\
D_{x} x_{n, m}^{2}=2 x_{n, m}+\frac{h_{n, m}^{y}\left(h_{n, m}^{x}\right)^{2}-\sigma_{n, m}^{y}\left(\sigma_{n, m}^{x}\right)^{2}}{h_{n, m}^{y} h_{n, m}^{x}-\sigma_{n, m}^{y} \sigma_{n, m}^{x}}=2 x_{n, m}+\Delta_{x x}^{x}, \\
D_{x} x_{n, m} y_{n, m}=y_{n, m}+\frac{h_{n, m}^{y} \sigma_{n, m}^{y}\left(h_{n, m}^{x}-\sigma_{n, m}^{x}\right)}{h_{n, m}^{y} h_{n, m}^{x}-\sigma_{n, m}^{y} \sigma_{n, m}^{x}}=y_{n, m}+\Delta_{x y}^{x}, \\
D_{x} y_{n, m}^{2}=-\frac{h_{n, m}^{y} \sigma_{n, m}^{y}\left(h_{n, m}^{y}-\sigma_{n, m}^{y}\right)}{h_{n, m}^{y} h_{n, m}^{x}-\sigma_{n, m}^{y} \sigma_{n, m}^{x}}=\Delta_{y y}^{x}, \\
D_{y} x_{n, m}^{2}=-\frac{h_{n, m}^{x} \sigma_{n, m}^{x}\left(h_{n, m}^{x}-\sigma_{n, m}^{x}\right)}{h_{n, m}^{y} h_{n, m}^{x}-\sigma_{n, m}^{y} \sigma_{n, m}^{x}}=\Delta_{x x}^{y}, \\
D_{y} x_{n, m} y_{n, m}=x_{n, m}+\frac{h_{n, m}^{x} \sigma_{n, m}^{x}\left(h_{n, m}^{y}-\sigma_{n, m}^{y}\right)}{h_{n, m}^{y} h_{n, m}^{x}-\sigma_{n, m}^{y} \sigma_{n, m}^{x}}=x_{n, m}+\Delta_{x y}^{y}, \\
D_{y} y_{n, m}^{2}=2 y_{n, m}+\frac{h_{n, m}^{x}\left(h_{n, m}^{y}\right)^{2}-\sigma_{n, m}^{x}\left(\sigma_{n, m}^{y}\right)^{2}}{h_{n, m}^{y} h_{n, m}^{x}-\sigma_{n, m}^{y} \sigma_{n, m}^{x}}=2 y_{n, m}+\Delta_{y y}^{y} .
\end{gathered}
$$

where the quantities $\Delta_{x x}^{x}, \Delta_{x y}^{x}, \Delta_{y y}^{x}, \Delta_{x x}^{y}, \Delta_{x y}^{y}$ and $\Delta_{y y}^{y}$ go to zero in the continuous limit when $h$ and $\sigma$ go to zero.

\section{EXAmple: the Potential Burgers EQUATion}

The Burgers equation

$$
u_{t}=\nu u_{x x}+u u_{x}
$$

a very well known partial differential equation, appears as a simplification of the Navier-Stokes equation and has been studied from many, if not all, points of view 4. It was proposed as a model for a viscous fluid, with a viscosity parameter $\nu$. When the viscosity parameter $\nu$ is set equal to zero, the Burgers equation degenerates into a quasilinear first order equation which is the prototype of a class of equations which presents nonlinear phenomena such as shock waves. In fact, the limit $\nu \rightarrow 0$ allows the study of these shock wave solutions as limits of the solutions of the viscous Burgers equation. In particular, although the inviscid Burgers equation has an infinite dimensional group of symmetries (being a first order equation), the limit of the symmetry group of the viscous Burgers equations provides a subgroup of the whole group of symmetries of the inviscid Burgers equation which is a useful tool in the study of the equation and in particular its discretization using invariant techniques.

Several invariant discretization approaches have been proposed to construct explicit numerical schemes for finding numerical solutions on invariant lattices [1, 10]. In some of these works, explicit comparison has been made, showing the higher accuracy and stability of these methods [5].

It is not our intention in this paper to present this kind of numerical results but rather to prove the possibility of constructing in an easy way such invariant discrete schemes and discuss the properties of a lattice satisfying the Clairaut-Schwarz-Young theorem from the numerical point of view. For the control of the numerical calculations we will study the time evolution of the initial condition provided by exact solutions of the Burgers equation. To simplify the presentation and with no loss of generality we will go over to consider the potential Burgers equation as this is point transformable into the linear heat equation for which many exact solutions are known.

Let us construct using the formulas introduced in the previous section the discrete scheme which preserves the point symmetries of the potential Burgers equation

$$
u_{y}-u_{x x}-u_{x}^{2}=0 .
$$

The point symmetries of 19 are [18]

$$
\begin{gathered}
\hat{V}_{1}=\partial_{x}, \quad \hat{V}_{2}=\partial_{y}, \quad \hat{V}_{3}=\partial_{u}, \quad \hat{V}_{4}=x \partial_{x}+2 y \partial_{y}, \quad \hat{V}_{5}=2 y \partial_{x}-x \partial_{u}, \\
\hat{V}_{6}=4 y x \partial_{x}+4 y^{2} \partial_{y}-\left(x^{2}+2 y\right) \partial_{u}, \quad \hat{V}_{\alpha}=\alpha(x, y) e^{-u} \partial_{u},
\end{gathered}
$$

where the function $\alpha(x, y)$ satisfies the heat equation

$$
\alpha_{y}=\alpha_{x x}
$$


The infinitesimal generator $\hat{V}_{\alpha}$ is the one responsible for the linearizability of the potential Burgers equation as it provides its linearizing transformation

$$
\alpha=e^{u}
$$

A function $F\left(x, y, u, u_{x}, u_{y}, u_{x x}\right)$ is invariant under the infinitesimal generators $\hat{V}_{1}, \hat{V}_{2}, \hat{V}_{3} \hat{V}_{4}, \hat{V}_{5}$ if it depends on

$$
I^{(1)}=\frac{u_{y}-u_{x}^{2}}{u_{x x}}
$$

It is then easy to see that 23 is weakly invariant also under $\hat{V}_{6}$ and $\hat{V}_{\alpha}$, i.e.,

$$
\begin{gathered}
\operatorname{pr}^{(2)} \hat{V}_{6} I^{(1)}=\frac{2}{u_{x x}}\left(I^{(1)}-1\right), \\
\operatorname{pr}^{(2)} \hat{V}_{\alpha} I^{(1)}=-\frac{e^{-u}}{u_{x x}}\left(\alpha u_{x}^{2}+2 \alpha_{x} u_{x}+\alpha_{y}\right)\left(I^{(1)}-1\right) .
\end{gathered}
$$

The potential Burgers equation $(19)$ is then given by

$$
I^{(1)}=1
$$

Taking into account the discrete prolongation (14) and the definition of the infinitesimal coefficients 15 we can construct the discrete prolongation of the vector fields $(20)$. It is easy to show that the conditions $(16)$ are satisfied for all symmetries 20 except for $\hat{V}_{6}$ for which

$$
\xi_{n, m+1}-\xi_{n, m}-\xi_{n+1, m+1}+\xi_{n+1, m}=-4\left[h_{n, m}^{x} h_{n, m}^{y}+\sigma_{n, m}^{x} \sigma_{n, m}^{y}\right] \neq 0
$$

and

$$
\tau_{n, m+1}-\tau_{n, m}-\tau_{n+1, m+1}+\tau_{n+1, m}=-8 h_{n, m}^{x} \sigma_{n, m}^{x} \neq 0
$$

Since we are presently interested in analyzing the role of the Schwarz condition on the construction of lattices and its consequences in numerical computations, we will not consider the symmetry with generator $\hat{V}_{6}$ in the following, that is, we restrict ourselves to a sugbroup of the whole symmetry group (note that we have also disregard in our analysis the operator $\hat{V}_{\alpha}$, which, as we have remarked above, is related to the linearization of the equation under study).

Taking into account (17) we have:

$$
\begin{gathered}
\operatorname{pr}^{d} \hat{V}_{1}=\partial_{x_{n, m}}, \quad \operatorname{pr}^{d} \hat{V}_{2}=\partial_{y_{n, m}}, \quad \operatorname{pr}^{d} \hat{V}_{3}=\partial_{u_{n, m}}, \\
\operatorname{pr}^{d} \hat{V}_{4}=x_{n, m} \partial_{x_{n, m}}+2 y_{n, m} \partial_{y_{n, m}}+h_{n, m}^{x} \partial_{h_{n, m}^{x}}+h_{n+1, m}^{x} \partial_{h_{n+1, m}^{x}}+h_{n, m+1}^{x} \partial_{h_{n, m+1}^{x}}+\sigma_{n, m}^{x} \partial_{\sigma_{n, m}^{x}} \\
+\sigma_{n, m+1}^{x} \partial_{\sigma_{n, m+1}^{x}}+2\left(h_{n, m}^{y} \partial_{h_{n, m}^{y}}+h_{n+1, m}^{y} \partial_{h_{n+1, m}^{y}}+h_{n, m+1}^{y} \partial_{h_{n, m+1}^{y}}+\sigma_{n, m}^{y} \partial_{\sigma_{n, m}^{y}}+\sigma_{n+1, m}^{y} \partial_{\sigma_{n+1, m}^{y}}\right) \\
-D_{x} u_{n, m} \partial_{D_{x} u_{n, m}}-2 D_{y} u_{n, m} \partial_{D_{y} u_{n, m}}-2\left[D_{x}\right]^{2} u_{n, m} \partial_{D_{x}^{2} u_{n, m}}, \\
\operatorname{pr}^{d} \hat{V}_{5}=2 y_{n, m} \partial_{x_{n, m}}-x_{n, m} \partial u_{n, m}+2 \sigma_{n, m}^{y} \partial_{h_{n, m}^{x}}+2 \sigma_{n+1, m}^{y} \partial_{h_{n+1, m}^{x}}+2\left(h_{n+1, m}^{y}+\sigma_{n, m}^{y}-h_{n, m}^{y}\right) \partial_{h_{n, m+1}^{x}} \\
+2 h_{n, m}^{y} \partial_{\sigma_{n, m}^{x}}+2 h_{n, m+1}^{y} \partial_{\sigma_{n, m+1}^{x}}-\partial_{D_{x} u_{n, m}}-2 D_{x} u_{n, m} \partial_{D_{y} u_{n, m}} .
\end{gathered}
$$

The commutation table of this algebra appears in Table 1 (it is, obviously the same as in the continuous case).

It is immediate to see that a discrete potential Burgers equation preserving the Lie algebra of $(19)$ given by the generators $\hat{V}_{1}, \hat{V}_{2}, \hat{V}_{3} \hat{V}_{4}, \hat{V}_{5}$ is:

$$
\mathcal{I}^{(1)}=\frac{\left[D_{x}\right]^{2} u_{n, m}}{D_{y} u_{n, m}-\left(D_{x} u_{n, m}\right)^{2}}=1 \text {, i.e., } \quad D_{y} u_{n, m}-\left[D_{x}\right]^{2} u_{n, m}-\left(D_{x} u_{n, m}\right)^{2}=0 .
$$

The continuous limit of 26$)$ is trivially given by $(19)$ when $h^{x}, h^{y}, \sigma^{x}$ and $\sigma^{y}$ go to zero preserving the structure of the lattice. Eq. (26) involves 6 lattice points centered around $(n, m)$, i.e., $(n, m),(n+1, m),(n, m+1)$, $(n+2, m),(n+1, m+1),(n, m+2)$. It explicitly reads:

$$
\begin{gathered}
\frac{-\sigma_{m}^{x}\left(u_{n+1, m}-u_{n, m}\right)+h_{n}^{x}\left(u_{n, m+1}-u_{n, m}\right)}{h_{n}^{x} h_{m}^{y}-\sigma_{m}^{x} \sigma_{n}^{y}}-\left[h _ { m } ^ { y } \left(\frac{h_{m}^{y}\left(u_{n+2, m}-u_{n+1, m}\right)-\sigma_{n+1}^{y}\left(u_{n+1, m+1}-u_{n+1, m}\right)}{h_{n+1}^{x} h_{m}^{y}-\sigma_{m}^{x} \sigma_{n+1}^{y}}\right.\right. \\
\left.-\frac{h_{m}^{y}\left(u_{n+1, m}-u_{n, m}\right)-\sigma_{n}^{y}\left(u_{n, m+1}-u_{n, m}\right)}{h_{n}^{x} h_{m}^{y}-\sigma_{m}^{x} \sigma_{n}^{y}}\right)-\sigma_{n}^{y}\left(\frac{h_{m+1}^{y}\left(u_{n+1, m+1}-u_{n, m+1}\right)-\sigma_{n}^{y}\left(u_{n, m+2}-u_{n, m+1}\right)}{h_{n}^{x} h_{m+1}^{y}-\sigma_{m+1}^{x} \sigma_{n}^{y}}\right. \\
\left.\left.-\frac{h_{m}^{y}\left(u_{n+1, m}-u_{n, m}\right)-\sigma_{n}^{y}\left(u_{n, m+1}-u_{n, m}\right)}{h_{n}^{x} h_{m}^{y}-\sigma_{m}^{x} \sigma_{n}^{y}}\right)\right]\left(h_{n}^{x} h_{m}^{y}-\sigma_{m}^{x} \sigma_{n}^{y}\right)^{-1} \\
-\frac{\left(h_{m}^{y}\left(u_{n+1, m}-u_{n, m}\right)-\sigma_{n}^{y}\left(u_{n, m+1}-u_{n, m}\right)\right)^{2}}{\left(h_{n}^{x} h_{m}^{y}-\sigma_{m}^{x} \sigma_{n}^{y}\right)^{2}}=0 .
\end{gathered}
$$




\begin{tabular}{c|ccccc} 
& $\hat{V}_{1}$ & $\hat{V}_{2}$ & $\hat{V}_{3}$ & $\hat{V}_{4}$ & $\hat{V}_{5}$ \\
\hline$\hat{V}_{1}$ & 0 & 0 & 0 & $\hat{V}_{1}$ & $-\hat{V}_{3}$ \\
$\hat{V}_{2}$ & 0 & 0 & 0 & $2 \hat{V}_{2}$ & $2 \hat{V}_{1}$ \\
$\hat{V}_{3}$ & 0 & 0 & 0 & 0 & 0 \\
$\hat{V}_{4}$ & $-\hat{V}_{1}$ & $-2 \hat{V}_{2}$ & 0 & 0 & $\hat{V}_{5}$ \\
$\hat{V}_{5}$ & $\hat{V}_{3}$ & $-2 \hat{V}_{1}$ & 0 & $-\hat{V}_{5}$ & 0
\end{tabular}

TABLE 1. Commutation table of the discrete invariance algebra.

To complete the difference scheme we have to associate to it a lattice equation which preserve the symmetries 25) or part of them. The complete list of discrete invariant of 25), obtained as usual, as solutions of the equations $\operatorname{pr}^{d} \hat{V}_{i}(\mathcal{K})=0$, are:

$$
\begin{gathered}
\mathcal{K}_{1}=\frac{h_{n+1, m}^{y}}{h_{n, m}^{y}}, \quad \mathcal{K}_{2}=\frac{h_{n, m+1}^{y}}{h_{n, m}^{y}}, \quad \mathcal{K}_{3}=\frac{\sigma_{n, m}^{y}}{h_{n, m}^{y}}, \quad \mathcal{K}_{4}=\frac{\sigma_{n+1, m}^{y}}{h_{n, m}^{y}}, \\
\mathcal{K}_{5}=\frac{1}{\left(h_{n, m}^{y}\right)^{3 / 2}}\left(h_{n, m}^{x} h_{n, m}^{y}-\sigma_{n, m}^{x} \sigma_{n, m}^{y}\right), \quad \mathcal{K}_{6}=\frac{1}{\left(h_{n, m}^{y}\right)^{3 / 2}}\left(h_{n, m+1}^{y} \sigma_{n, m}^{x}-h_{n, m}^{y} \sigma_{n, m+1}^{x}\right), \\
\mathcal{K}_{7}=\frac{1}{\left(h_{n, m}^{y}\right)^{3 / 2}}\left(h_{n, m}^{x}\left(h_{n+1, m}^{y}-h_{n, m}^{y}\right)-\sigma_{n, m}^{y}\left(h_{n, m+1}^{x}-h_{n, m}^{x}\right)\right), \\
\mathcal{K}_{8}=\frac{1}{\left(h_{n, m}^{y}\right)^{3 / 2}}\left(h_{n, m}^{x} \sigma_{n+1, m}^{y}-h_{n+1, m}^{x} \sigma_{n, m}^{y}\right), \quad \mathcal{K}_{9}=\frac{1}{\left(h_{n, m}^{y}\right)^{1 / 2}}\left(h_{n, m}^{x}+2 \sigma_{n, m}^{y} D_{x} u_{n, m}\right), \\
\mathcal{K}_{10}=\frac{D_{y} u_{n, m}-\left(D_{x} u_{n, m}\right)^{2}}{\left[D_{x}\right]^{2} u_{n, m}} .
\end{gathered}
$$

Our first choice for the difference scheme is an orthogonal cartesian lattice given, in the coordinates $h_{n, m}$ and $\sigma_{n, m}$ by

$$
h_{n, m}^{y}=b, \quad \sigma_{n, m}^{y}=0, \quad h_{n, m}^{x}=a, \quad \sigma_{n, m}^{x}=0,
$$

where $a$ and $b$ are arbitrary constants, which in the continuous limit go to zero. This lattice, which is clearly invariant under all the symmetries which satisfy the commutativity constraint, corresponds to the Lie point symmetry infinitesimal generators

$$
\xi_{n, m}=\tau_{n, m}=1
$$

These generators comply with the constraints in 16 and the orthogonal lattice 29 satisfies the ClairautSchwarz-Young theorem.

To carry out a comparison between this invariant Schwarzian lattice with other lattices which lack these properties, we will consider an exponential non Schwarzian lattice as given in [14] by

$$
y_{n, m}=b m+b_{0}, \quad x_{n, m}=(1+c)^{m}\left(a n+a_{0}\right),
$$

where $a, a_{0}, b, b_{0}$ and $c$ are arbitrary constants. $b$ and $a$ are the lattice spacing and $c$ is a dilation parameter which, when set equal to zero reduces this lattice to an orthogonal lattice. This lattice corresponds to the Lie point symmetry infinitesimal generators

$$
\xi_{n, m}=(1+c)^{m}\left(k_{1} n+k_{2}\right)+k_{0} x_{n, m}, \quad \tau_{n, m}=k_{3}
$$

which clearly do not satisfy 16 . The non Schwarzian property of this lattice can also be seen by considering the lattice differences

$$
h_{n, m}^{y}=b, \quad \sigma_{n, m}^{y}=0, \quad h_{n, m}^{x}=(1+c)^{m} a, \quad \sigma_{n, m}^{x}=c(1+c)^{m} a n .
$$

and comparing them with (11). This non Schwarzian lattice is not invariant under the potential Burgers symmetry group $20 ; ; \mathcal{K}_{5}, \mathcal{K}_{6}, \mathcal{K}_{7}$ and $\mathcal{K}_{9}$ are non constants.

In the following Section we will provide a numerical test about the possible importance of the ClairautSchwarz-Young theorem, by studying numerically the evolution provided by the symmetry preserving discretized potential Burgers equation (27) on the two different lattices we introduced above, i.e., the Schwarzian and non Schwarzian lattices 29,33$)$.

\section{Numerical Calculation Results for the Discrete potential Burgers EQUATION}

In order to compare the precision and accuracy of different lattices used to compute numerically the solution of the potential Burgers equation, we will construct two of its exact solutions (associated to different symmetries 
of the heat equation) and use them as initial conditions for the evolution of the Burgers map (27). In fact, we will not do a direct comparison of the evolution of this map in the two lattices (29) and (33) but compare the solution of the map in each lattice with the exact solution of the continuous equation.

We introduce two invariant solutions of (21) and transform them into solutions of the potential Burgers equation [14] using (22). Starting from the traveling wave solution of the heat equation (invariant under a combination of $x$ and $y$ translations) we get as a simple solution, bounded for $x \in \mathbf{R}^{+}$for each $y$,

$$
f_{1}(x, y)=\log \left(1+e^{-(x-y)}\right) .
$$

A second exact solution (the fundamental solution) is given by the Galilei-invariant solution of the heat equation

$$
f_{2}(x, y)=\log \left(1+\frac{e^{-x^{2} /(4 y)}}{\sqrt{y}}\right) \text {. }
$$

To compare the results given by the evolution of the map on a given lattice to the evolution given by the exact solution we introduce a global estimator, the usual (relative) distance in the discrete analog of the $L^{2}$ space:

$$
\chi_{\text {lattice }}(f)=\sqrt{\frac{\sum_{n, m}\left(f_{n, m}^{\text {lattice }}-f_{n, m}\right)^{2}}{\sum_{n, m} f_{n, m}^{2}}}
$$

where $f_{n, m}$ and $f_{n, m}^{\text {lattice }}$ are the values of the exact solution and the numerical one, respectively, computed in the points of the lattices 29,33 .

Since our intention is to compare the lattices, we will not insist on improving the precision and accuracy of the solution by modifying in an optimal way the parameters involved in the computation. For the orthogonal lattice we will take (for $f_{1}$ and $f_{2}$ ) $a=b=0.1$ in a square $\mathcal{D}$ of $8 \times 8$ points in the $x, y$ plane. We will use the same number of points in all the cases we will consider, in order to keep as far as possible the same round off errors due to machine precision. Augmenting the number of points enlarges the numerical instabilities which could be reduced by increasing the precision of the calculations at the cost of the time of calculus. These instabilities are non-physical and we decide to avoid them by reducing appropriately the number of points. The region covered by the lattice in the two cases (orthogonal and exponential) can be different for the same lattice spacing. See for example in Figure 1 the cases (1) and (2). In the exponential lattice we will consider two different situations: (i) the spacing is the same as in the orthogonal case $(a=b=0.1)$, and thus for the exponential lattice the region is deformed and enlarged with respect to the square $\mathcal{D}$ considered in the orthogonal lattice, (ii) we will modify $a$ in such a way that the 64 points of the lattice are inside the square $\mathcal{D}$. Let us notice that in this case part of $\mathcal{D}$ is not covered by the lattice, and this may create problems as we will see later.

The exponential lattice has a parameter $c$ controlling the dilation of the $x$ variable. As we said above, when $c=0$ the lattice is orthogonal. We have considered in these numerical calculations two cases, $c=0.1$ and $c=0.15$, to compare the different behavior of the exponential lattice when it turns into an orthogonal one. In these two cases, the parameter $a$ is taken as $a=0.0375$ and $a=0.0513$ respectively, when we keep the 64 point of the lattice inside $\mathcal{D}$ (see Figure 1 for a graphical description of the four lattices under study).

The $\chi$ estimator is given in Table 2 and gives raise to the following conclusions:

(1.) The orthogonal lattice (Schwarzian lattice) provides better results than the exponential one (non Schwarzian lattice) in all cases except one.

(2.) The results for the exponential lattice with different values of $c$ show that the approximation is better when the lattice is closer to a Schwarzian lattice (recall that when $c \rightarrow 0$ the exponential lattice becomes orthogonal), for the two solutions considered.

(3.) The value of the $\chi$ estimator for the traveling wave solution in the exponential lattice when $a=0.0513$ and $c=0.1$ is lower than the corresponding value for the orthogonal case. In this case (Figure 13 ) the lattice is close to the orthogonal one but there is a region in $\mathcal{D}$ which is not covered by the exponential lattice. This is the region where the round off errors the computer makes in calculating the points in the orthogonal lattice are greater. This is the reason for this unsatisfactory value. When $c=0.15$ the lattice is far from the orthogonal one and thus $\chi$ is greater than in the orthogonal case.

\section{Conclusions}

In this work we have shown that also in the case of partial difference equations we can introduce a set of variables which are in one to one correspondence with the grid points when we substitute them by the lattice differences and the derivatives on the lattice of the dependent function. This correspondence allows us to write down the invariance equations by using only the knowledge of the continuous invariants. 


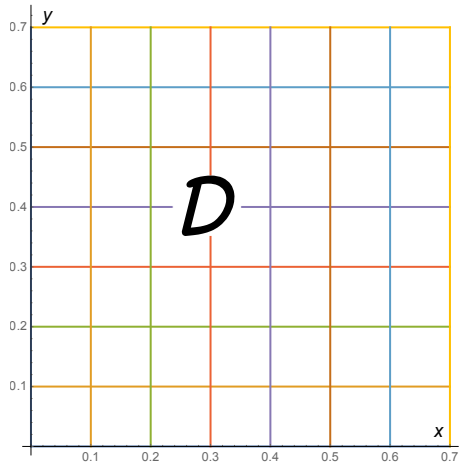

(1)

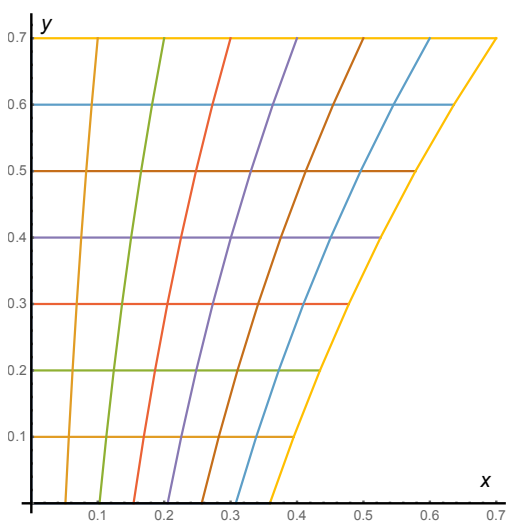

(3)

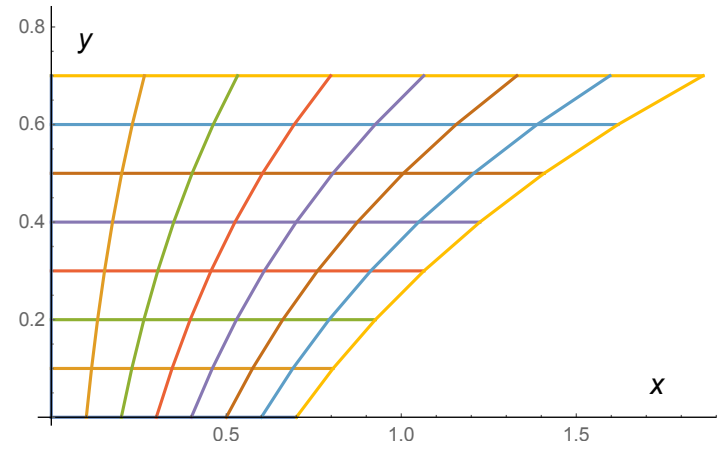

(2)

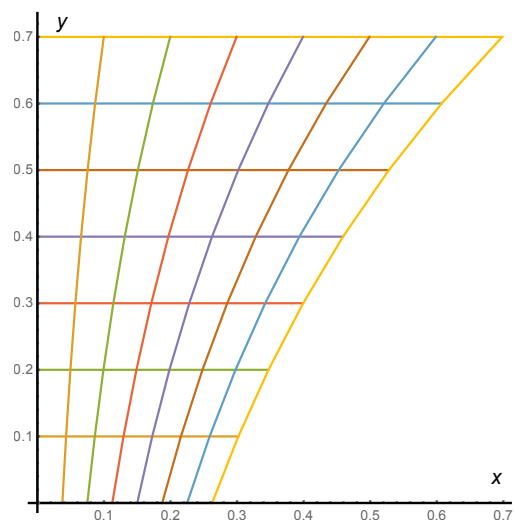

(4)

Figure 1. (1) Orthogonal lattice: $a=0.1, b=0.1$. (2) Exponential lattice: $a=0.1, b=0.1, c=0.15$. (3) Exponential lattice: $a=0.0513, b=0.1, c=0.1$. (4) Exponential lattice: $a=0.0375, b=0.1, c=0.15$. Dimension: $8 \times 8$ points.

\begin{tabular}{lllllll}
\hline & \multicolumn{2}{c}{$\chi_{\text {ort }}$} & \multicolumn{2}{c}{$\chi_{\exp }, c=0.1$} & \multicolumn{2}{c}{$\chi_{\exp }, c=0.15$} \\
\hline$f_{1}$ & $(1)$ & 0.01267 & $(5)$ & 0.01437 & $(2)$ & 0.01651 \\
& & & $(3)$ & 0.01147 & $(4)$ & 0.01408 \\
\hline$f_{2}$ & $(1)$ & 0.00249 & $(5)$ & 0.00430 & $(2)$ & 0.00610 \\
& & & $(3)$ & 0.00642 & $(4)$ & 0.00913 \\
\hline
\end{tabular}

TABLE 2. $\chi$ estimator values for the functions $f_{1}$ and $f_{2}$ given by 34 and 35 , respectively. (1): $a=0.1$, lattice (1) in Figure 1 (2): $a=0.1$, lattice (2) in Figure 1. (3): $a=0.0513$, lattice (3) in Figure 1 (4): $a=0.0375$, lattice (4) in Figure 1. (5): $a=0.1$, the exponential lattice is similar to the lattice (2) in Figure 1

The numerical calculation we carried out in Section 4 shows that the Schwarzian property seems important in providing better numerical results.

More examples should be done, both to understand the stability of the models we construct in this way and the analysis of the lattices in the Schwarzian and non Schwarzian case. There might be boundary value problems where a non Schwarzian lattice adapted to the geometry of the problem could be better than a Schwarzian one.

\section{ACKNOWLEDGEMENTS}

DL has been partly supported by the Italian Ministry of Education and Research, 2010 PRIN Continuous and discrete nonlinear integrable evolutions: from water waves to symplectic maps and by INFN IS-CSN4 Mathematical Methods of Nonlinear Physics. DL thanks the Departamento de Física Teórica II of the Complutense University in Madrid for its hospitality. MAR was supported by the Spanish MINECO under project FIS2015-63966. The authors would like to thank the referees for their useful comments.

\section{REFERENCES}

[1] Bihlo A and Nave J-C 2014 Convecting reference frames and invariant numerical models J. Comput. Phys. 272 656-663; DOI:10.1016/j.jcp.2014.04.042

[2] Bourlioux A, Cyr-Gagnon C, and Winternitz P 2006 Difference schemes with point symmetries and their numerical tests J. Phys. A: Math. Gen. 39 6877-6896; DOI:10.1088/0305-4470/39/22/006 
[3] Bourlioux A, Rebelo R, and Winternitz P 2008 Symmetry preserving discretization of SL(2,R) invariant equations J. Nonlinear Math. Phys. 15 (Suppl.3) 362-372

[4] Burgers J M 1974 The Nonlinear Diffusion Equation, Asymptotic Solutions and Statistical Problems, Springer, Dordrecht.

[5] Chhay M and Hamdouni A 2010 A new construction for invariant numerical schemes using moving frames C.R. Mecanique 338 97-101; DOI:10.1016/j.crme.2010.01.001

[6] Dorodnitsyn V A 1991 Transformation groups in difference spaces, J. Soviet Math. 55 1490-1517

[7] Dorodnitsyn V A 2011 Applications of Lie Groups to Difference Equations, CRC Press, Boca Raton

[8] Dorodnitsyn V A and Winternitz P 2000 Lie Point Symmetry Preserving Discretizations for Variable Coefficient Korteweg-de Vries Equations Nonlinear Dynamics 22 49-59

[9] Kim P and Olver P J 2004 Geometric integration via multi-space Regular and Chaotic Dynamics 9 213-226

[10] Kim P 2008 Invariantization of the Crank-Nicolson method for Burgers' equation Physica D 237 243-254; DOI:10.1016/j.physd.2007.09.001

[11] Levi D, Olver P, Thomova Z and Winternitz P (editors) 2011 Symmetries and Integrability of Difference Equations, CUP, LMS Lecture Series.

[12] Levi D, Scimiterna C, Thomova Z and Winternitz P 2012 Contact transformations for difference schemes J. Phys. A: Math. Theor. 45 022001; DOI:10.1088/1751-8113/45/2/022001

[13] Levi D, Thomova Z and Winternitz P 2011 Are there contact transformations for discrete equations? J. Phys. A: Math. Theor. 44 265201; DOI:10.1088/1751-8113/44/26/265201

[14] Levi D and Rodríguez M A 2013 On the construction of partial difference schemes I: The Clairaut, Schwarz, Young theorem on the lattice, J. Phys. A: Math. Theor. 46 295203; DOI:10.1088/1751-8113/46/29/295203

[15] Levi D and Winternitz P 1991 Continuous symmetries of discrete equations, Phys. Lett. A 152 335-338; DOI:10.1016/0375-9601(91)90733-O

[16] Levi D and Winternitz P 1996 Symmetries of discrete dynamical systems. J.Math. Phys. 37 5551-5576; DOI:10.1063/1.531722

[17] Levi D and Winternitz P 2006 Continuous symmetries of difference equations J. Phys. A: Math. Gen. 39 R1; DOI:10.1088/0305-4470/39/2/R01

[18] Olver P J 1993 Applications of Lie Groups to Differential Equations, Springer-Verlag, New York

[19] Rebelo R and Valiquette F 2013 Symmetry preserving numerical schemes for partial differential equations and their numerical tests J. Difference Eq. Appl. 19 737-757; DOI:10.1080/10236198.2012.685470

[20] Rebelo R and Valiquette F 2015 Invariant Discretization of Partial Differential Equations Admitting Infinite-Dimensional Symmetry Groups J. Difference Eq. Appl. bf 21 285-318; DOI:10.1080/10236198.2015.1007134

[21] Rebelo R and Winternitz P 2009 Invariant difference schemes and their applications to SL(2,R) invariant differential equations J. Phys. A: Math. Theor. 42 454016; DOI:10.1088/1751-8113/42/45/454016

[22] Winternitz P 2004 Symmetries of discrete systems. Discrete Integrable Systems, vol. 644, Lecture Notes in Physics. Ed B Grammaticos, Y Kosmann-Schwarzbach, and T Tamizhmani (Berlin, Springer Verlag) pp 185-243. See also arXiv:nlin.SI/0309058

[23] Winternitz P 2011 Symmetry preserving discretization of differential equations and Lie point symmetries of differential-difference equations. Symmetries and Integrability of Difference Equations. LMS Lecture Series. Ed D Levi, P J Olver, Z Thomova and P Winternitz. 